\section{SAT0631 LACK OF ASSOCIATION BETWEEN CLINICAL AND ULTRASOUND MEASURES OF DISEASE ACTIVITY IN RHEUMATOID ARTHRITISCLINICAL REMISSION: A CROSS-SECTIONAL ANALYSIS}

K.F. Baker, B. Thompson, D. Lendrem, A.G. Pratt, J.D. Isaacs. Musculoskeletal Research Group, Newcastle NIHR Biomedical Research Centre, Newcastle University and Newcastle upon Tyne Hospitals NHS Foundation Trust, Newcastle upon Tyne, UK

Background: Ultrasound (US) measures of synovitis and tenosynovitis have been shown to persist in patients with rheumatoid arthritis (RA) who achieve clinical remission.

Objectives: To assess the prevalence of such US findings in RA clinical remission, and analyse whether the US abnormalities found in this group of patients could be predicted by their clinical parameters.

Methods: Patients with established RA (1987 or 2010 diagnostic criteria) in clinical remission (DAS28-CRP<2.4) on conventional synthetic disease-modifying anti-rheumatic drugs (DMARDs) were recruited as part of the Biomarkers of Remission in Rheumatoid Arthritis (BioRRA) Study. Patients who had received systemic glucocorticoids in the past 3 months were excluded. All patients underwent baseline clinical assessment followed by a 7-joint US scan (Backhaus et al). US assessment was performed by the same operator (KB) blinded to disease activity score. Synovial Power Doppler (SPD) and greyscale (SGS) change were measured using a 4-point semi-quantitative scale (0-3); tendon GS (TGS) and erosions were classified as either present ${ }^{1}$ or absent (0). Intra- and inter-rater scoring agreement was good (Cohen's kappa 0.72 and 0.61 respectively). The association between individual dependent variables (SPD, SGS, TGS, and erosions) and clinical parameters was assessed by multivariate ordinal logistic regression, with Benjamini-Hochberg adjustment for multiple testing.

Results: 66 patients with RA in remission (median disease duration 6 years, median age 66 years) were included in the analysis. US abnormalities were common in both DAS28-CRP and ACR/EULAR 2011 Boolean remission (table 1). Seven associations were significant at the unadjusted $p<0.05$ level (figure 1). Only two associations remained statistically significant after multiple test correction, namely those of disease duration and TJC with higher and lower erosion scores respectively (table 2).

Abstract SAT0631 - Table 1

\begin{tabular}{lcccc}
\hline Criterion & $\begin{array}{c}\text { US } \\
\text { parameter }\end{array}$ & \multicolumn{2}{c}{ Remission Definition } & $\mathrm{p}\left(\chi^{2}\right)$ \\
\cline { 3 - 5 } & & $\begin{array}{l}\text { DAS28- } \\
\text { CRP }<2.4 \\
(\mathrm{n}=66)\end{array}$ & $\begin{array}{c}\text { ACR/EULAR } \\
\text { Boolean } \\
(\mathrm{n}=40)\end{array}$ & \\
\hline $\mathrm{n}(\%)$ patients with total & SPD & $17(26)$ & $10(25)$ & 0.931 \\
score $\geq 1$ & SGS & $66(100)$ & $40(100)$ & $\mathrm{n} / \mathrm{a}$ \\
& TGS & $29(44)$ & $17(43)$ & 0.885 \\
& Erosions & $45(68)$ & $25(63)$ & 0.549 \\
$\mathrm{n}(\%)$ patients with any joint & SPD & $8(12)$ & $6(15)$ & 0.671 \\
score $\geq 2$ & SGS & $48(73)$ & $27(68)$ & 0.566 \\
\hline
\end{tabular}

Abstract SAT0631 - Table 2.

\begin{tabular}{|c|c|c|c|c|c|}
\hline $\begin{array}{l}\text { US } \\
\text { score }\end{array}$ & $\begin{array}{c}\text { Clinical } \\
\text { parameter }\end{array}$ & $\begin{array}{l}\text { Odds ratio (OR) } \\
\text { increase in US } \\
\text { score }\end{array}$ & $95 \% \mathrm{Cl}$ & $\begin{array}{c}\text { Unadjusted } \\
\text { multivariate } p \\
\text { value }\end{array}$ & $\begin{array}{c}\text { Adjusted } \\
\text { multivariate } \mathrm{p} \\
\text { value }\end{array}$ \\
\hline \multirow[t]{2}{*}{ SGS } & Male sex & 5.04 & $\begin{array}{l}1.47- \\
17.26\end{array}$ & 0.010 & 0.139 \\
\hline & $\begin{array}{l}\text { ESR } \\
(\mathrm{mm} / \mathrm{hr})\end{array}$ & 1.05 & $\begin{array}{c}1.00- \\
1.09\end{array}$ & 0.038 & 0.245 \\
\hline \multirow[t]{2}{*}{ TGS } & SJC28 & 5.37 & $\begin{array}{l}1.46- \\
19.72\end{array}$ & 0.011 & 0.159 \\
\hline & $\begin{array}{l}\text { Alcohol } \\
\text { intake } \\
\text { (units/week) }\end{array}$ & 0.88 & $\begin{array}{c}0.77- \\
1.00\end{array}$ & 0.044 & 0.307 \\
\hline \multirow[t]{3}{*}{ Erosion } & $\begin{array}{c}\text { Disease } \\
\text { duration } \\
\text { (years) }\end{array}$ & 1.16 & $\begin{array}{l}1.06- \\
1.27\end{array}$ & 0.002 & 0.024 \\
\hline & TJC28 & 0.17 & $\begin{array}{c}0.05- \\
0.56\end{array}$ & 0.004 & 0.025 \\
\hline & $\begin{array}{l}\text { ESR } \\
(\mathrm{mm} / \mathrm{hr})\end{array}$ & 0.92 & $\begin{array}{c}0.85- \\
0.99\end{array}$ & 0.022 & 0.101 \\
\hline
\end{tabular}

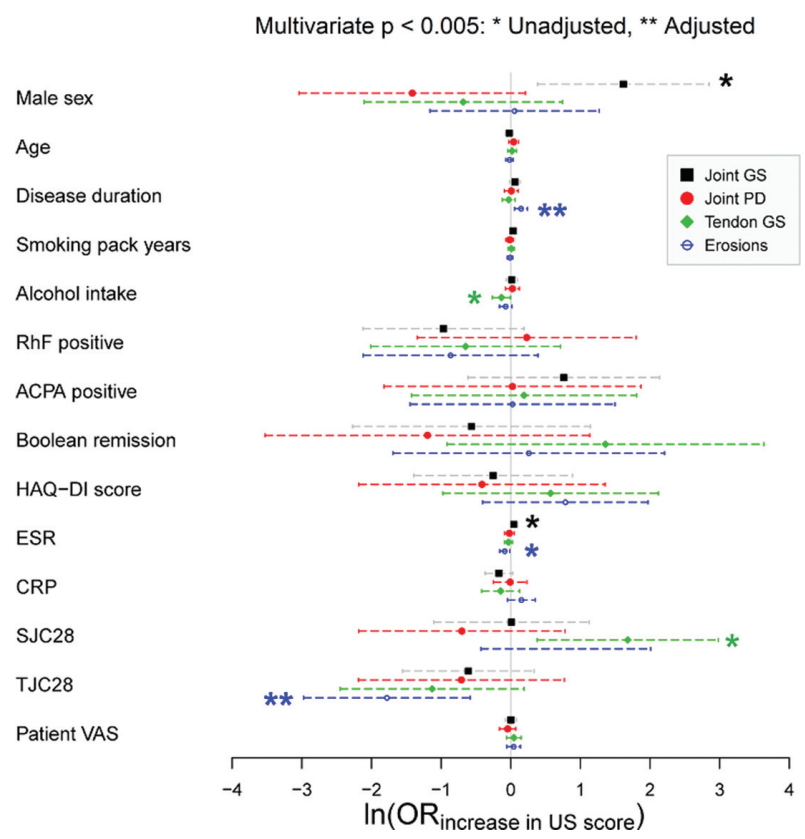

Conclusions: We demonstrate considerable subclinical US findings in RA patients in clinical remission, even when remission is defined using the more stringent ACR/EULAR Boolean criteria. Patients with longer disease duration and fewer tender joints had more joint erosions, though no other significant associations were seen after multiple test correction. Most strikingly SPD, which portends a poor prognosis, failed to show significant association with any of the clinical parameters. Our results suggest that clinical and ultrasound examinations may serve complementary, rather than duplicative, roles in the assessment of RA remission.

\section{REFERENCE:}

[1] Backhaus, et al. Arthritis Rheum;61:1194-201.

Disclosure of Interest: None declared

DOI: 10.1136/annrheumdis-2018-eular.1731

\section{SAT0632 ULTRASOUND IN THE MANAGEMENT OF RHEUMATOID ARTHRITIS USING A NOVEL PRAGMATIC ALGORITHM: A MULTICENTRIC OBSERVATIONAL STUDY}

K.R. Bonfiglioli ${ }^{1}$, R. Chakr ${ }^{2}$, R. Lima ${ }^{3}$, E. Paiva ${ }^{4}$, I. Moss ${ }^{5}$, R. Giorgi ${ }^{6}$, M. Rassi ${ }^{3}$, R. Cardoso ${ }^{3}$, F. Nadaf ${ }^{3}$, R. Santos ${ }^{3}$, D. Ribeiro ${ }^{3}$, L. Arruda ${ }^{3}$, E. Melo ${ }^{5}$, D. Furtado ${ }^{5}$, A. Funke ${ }^{4}$, N. Andrade ${ }^{2}$, M. Fantinel ${ }^{2}$, L. Rossi ${ }^{2}$, M. Resmini ${ }^{2}$, N. Sacilotto ${ }^{6}$, J. A. Mendonça ${ }^{7}$, I. Laurindo ${ }^{8} .{ }^{1}$ Reumathology, Hospital das Clinicas da Universidade de São Paulo, São Paulo; ${ }^{2}$ Hospital de Clínicas de Porto Alegre, Porto Alegre;

${ }^{3}$ Hospital de Base do Distrito Federal, Brasilia; ${ }^{4}$ Hospital de Clinicas de Curitiba, Curitiba; ${ }^{5}$ Hospital Universitário Clementino Fraga Filho, Rio de Janeiro; ${ }^{6}$ Instituto de Assistência Médica ao Servidor Público Estadual, São Paulo; ${ }^{7}$ PUC- Campinas, Campinas; ${ }^{8}$ Universidade Nove de Julho, São Paulo, Brazil

Background: Recently, novel algorithms for the pragmatic use of US in the management of RA patients were published in order to guide US use in various clinical scenarios.

Objectives: To evaluate the performance of the 2016 algorithm ${ }^{1}$ proposed for evaluation of therapeutic response and its potential to contribute in decisionmaking.

Methods: Multicentric (5 centres), cross-sectional and observational study. Inclusion criteria: Patients older than 18 years old, RA diagnosis (ACR/EULAR criteria), receiving stable doses of conventional synthetic disease-modifying antirheumatic drugs (csDMARDs) for the last six months. Exclusion criteria: use of biological DMARDs, severe articular deformities. Patients were submitted to clinical examination, CDAI(Clinical Disease Activity Index) was recorded and US examination performed by trained rheumatologists blinded to CDAI results. The following joints were examined: wrists, metacarpophalangeal ( 2 and 3$)$, proximal interphalangeal (2 and 3 ) and metatarsophalangeal ( 2 and 5 ), in addition to any symptomatic joint.Synovitis was determined according to OMERACT.Joint distension (grey scale, GS) and Power Doppler (PD) graded on semiquantitative scale: absent $=0$, mild $=1$, moderate $=2$, intense $=3$. Total individual GS and PD scores were calculated by the sum of each joint scores. Therefore, each participant had two separate scores (GS and PD) 